\title{
A Geometric Algorithm for Learning Oblique Decision Trees
}

\author{
Naresh Manwani and P.S. Sastry \\ Indian Institute of Science, Bangalore-12, India \\ $\{$ naresh, sastry\}@ee.iisc.ernet.in
}

\begin{abstract}
In this paper we present a novel algorithm for learning oblique decision trees. Most of the current decision tree algorithms rely on impurity measures to assess goodness of hyperplanes at each node. These impurity measures do not properly capture the geometric structures in the data. Motivated by this, our algorithm uses a strategy, based on some recent variants of SVM, to assess the hyperplanes in such a way that the geometric structure in the data is taken into account. We show through empirical studies that our method is effective.
\end{abstract}

\section{Introduction}

Decision tree is a well known and widely used method for classification and regression. The popularity of decision tree is because of its simplicity. In this paper we deal with oblique decision trees. These are binary decision trees where each non-leaf node is associated with a hyperplane (also called a split rule) and each leaf with a class label. To classify a pattern we follow a path in the tree by going down the left or right subtree at each node based on whether the pattern is on the positive or negative side of the hyperplane associated with the node; when we reach the leaf the pattern gets assigned the label of the leaf. Most approaches for learning decision trees are recursive algorithms for building the tree in a top down fashion. At each stage, we need to find a hyperplane to associate with the current node which is done by searching for a hyperplane which minimizes some impurity measure. Gini index and entropy are frequently used impurity measures. CART-LC [2, OC1 8] are few examples of decision tree approaches which learn oblique decision trees by optimizing such impurity measures. All decision tree algorithms employ greedy search and hence a hyperplane assigned to a node once, is never changed. If a bad hyperplane is learnt at some node, it can not be rectified anymore and the effect of it may be a large tree which, in turn, may lead to poor generalization error.

A problem with all impurity measures is that they depend only on the number of (training) patterns of either class on each side of the hyperplane. If we change the class regions without changing the effective areas of class regions on either side of the hyperplane, the impurity measure will not change. Thus the impurity measures do not really capture the geometric structure of class distributions. In this paper we present a new decision tree learning algorithm which is based

S. Chaudhury et al. (Eds.): PReMI 2009, LNCS 5909, pp. 25-31, 2009.

(C) Springer-Verlag Berlin Heidelberg 2009 
on the idea of capturing the geometric structure. For this we borrow ideas from some recent variants of the SVM method which are quite good at capturing (linear) geometric structure of the data. The Proximal SVM [5] finds two parallel clustering hyperplanes, one for each class, and takes the hyperplane situated in the middle of the two as the classifying hyperplane. However, often the points of the two classes are not clustered around parallel hyperplanes. Multisurface Proximal SVM [6] finds two clustering hyperplanes, one for each class, which are not necessary parallel, and the data is classified based on the nearness to the hyperplanes. Many times the class distribution is such that just one pair of hyperplanes is not good enough for classification. For such cases, they [6] extend this idea using the kernel trick of (effectively) learning the pair of hyperplanes in a high dimensional space to which the patterns are transformed.

Motivated by Proximal SVM, we derive our decision tree approach as follows. At each node, we use the idea of Multisurface Proximal SVM to find the linear tendencies in the data by learning hyperplanes around which the data of different classes are clustered. After finding these hyperplanes, the natural guess for a split rule at this node is the angle bisector of the two hyperplanes. This allows us to capture geometry of the classification boundary to some extent. Then, in the usual style of decision tree algorithms, we split the data based on this angle bisector and recursively learn the left and right subtrees of this node. Since, in general, there will be two angle bisectors, we select the one which is better based on an impurity measure. In this paper we discuss the algorithm for only two class classification problem. Since our decision tree approach is motivated by the geometry of the classification problem, we call it Geometric Decision Tree (Geometric DT).

\section{The Algorithm}

For a general decision tree algorithm, the main computational task is: given a set of data, find the best hyperplane to split the data. Let $S^{t}$ be the set of points falling at node $t$. Let $n_{+}^{t}$ and $n_{-}^{t}$ denote the number of patterns of the two classes (say, ' +1 ' and ' -1 ') at that node. Let $d$ be the data dimension. Let $A \in \Re^{n_{+}^{t} \times d}$ be the matrix representing points of class +1 as row vectors at node $t . A_{i}$ is the $i$ th row of $A$ which is a row vector in $\Re^{d}$. Similarly $B \in \Re^{n_{-}^{t} \times d}$ be the matrix whose rows contain points of class -1 at node $t$. Let $h_{1}: \mathbf{w}_{1}^{t} \mathbf{x}+b_{1}=0$ and $h_{2}: \mathbf{w}_{2}^{t} \mathbf{x}+$ $b_{2}=0$ be two clustering hyperplanes, one for each class. As mentioned before we use Multisurface Proximal SVM 6], also called Generalized Proximal SVM (GEPSVM), to find the two clustering hyperplanes. The hyperplane $h_{1}$ is closest to all points of class +1 and farthest from class -1 . Similarly the hyperplane $h_{2}$ is closest to all points of class -1 and farthest from class +1 . Then the optimization problems for finding $\left(\mathbf{w}_{1}, b_{1}\right)$ and $\left(\mathbf{w}_{2}, b_{2}\right)$ can be written as [6]

$$
\left(\mathbf{w}_{1}, b_{1}\right)=\operatorname{argmin}_{(\mathbf{w}, b) \neq 0} \frac{\left\|A \mathbf{w}+b \mathbf{e}_{n_{+}^{t}}\right\|^{2}}{\left\|B \mathbf{w}+b \mathbf{e}_{n_{-}^{t}}\right\|^{2}}
$$




$$
\left(\mathbf{w}_{2}, b_{2}\right)=\operatorname{argmin}_{(\mathbf{w}, b) \neq 0} \frac{\left\|B \mathbf{w}+b \mathbf{e}_{n_{-}^{t}}\right\|^{2}}{\left\|A \mathbf{w}+b \mathbf{e}_{n_{+}^{t}}\right\|^{2}}
$$

Where $\|$.$\| denotes the standard euclidean norm, \mathbf{e}_{n_{+}^{t}}$ is $\left(n_{+}^{t} \times 1\right)$ vector of ones and $\mathbf{e}_{n_{-}^{t}}$ is $\left(n_{-}^{t} \times 1\right)$ vector of ones. These optimization problems can be further reduced to 6 ]

$$
\begin{aligned}
& \tilde{\mathbf{w}}_{1}=\operatorname{argmin}_{\tilde{\mathbf{w}} \neq 0} \frac{\tilde{\mathbf{w}}^{T} G \tilde{\mathbf{w}}}{\tilde{\mathbf{w}}^{T} H \tilde{\mathbf{w}}} \\
& \tilde{\mathbf{w}}_{2}=\operatorname{argmin}_{\tilde{\mathbf{w}} \neq 0} \frac{\tilde{\mathbf{w}}^{T} H \tilde{\mathbf{w}}}{\tilde{\mathbf{w}}^{T} G \tilde{\mathbf{w}}}
\end{aligned}
$$

where $\tilde{\mathbf{w}}=(\mathbf{w}, b), G=\left[A \mathbf{e}_{n_{+}^{t}}\right]^{T}\left[A \mathbf{e}_{n_{+}^{t}}\right]$ and $H=\left[B \mathbf{e}_{n_{-}^{t}}\right]^{T}\left[B \mathbf{e}_{n_{-}^{t}}\right]$. This problem of finding the two clustering hyperplanes $\left(\mathbf{w}_{1}, b_{1}\right)$ and $\left(\mathbf{w}_{2}, b_{2}\right)$ gets reduced [6] to finding the eigenvectors corresponding to the maximum and minimum eigenvalue of the following generalized eigenvalue problem

$$
H \tilde{\mathbf{w}}=\gamma G \tilde{\mathbf{w}}
$$

Once we find these hyperplanes, the hyperplane we associate with the current node will be one of the angle bisectors of these two hyperplanes because it divide the space in such a way that on one side points of one class are clouded and on the other side points of second class are clouded. Let the hyperplanes $\mathbf{w}_{3}^{t} \mathbf{x}+b_{3}=0$ and $\mathbf{w}_{4}^{t} \mathbf{x}+b_{4}=0$ be the angle bisectors of $\mathbf{w}_{1}^{t} \mathbf{x}+b_{1}=0$ and $\mathbf{w}_{2}^{t} \mathbf{x}+b_{2}=0$, then

$$
\begin{aligned}
& \tilde{\mathbf{w}}_{3} \triangleq\left(\mathbf{w}_{3}, b_{3}\right)=\left(\frac{\mathbf{w}_{1}}{\left\|\mathbf{w}_{1}\right\|}-\frac{\mathbf{w}_{2}}{\left\|\mathbf{w}_{2}\right\|}, \frac{b_{1}}{\left\|\mathbf{w}_{1}\right\|}-\frac{b_{2}}{\left\|\mathbf{w}_{2}\right\|}\right) \\
& \tilde{\mathbf{w}}_{4} \triangleq\left(\mathbf{w}_{4}, b_{4}\right)=\left(\frac{\mathbf{w}_{1}}{\left\|\mathbf{w}_{1}\right\|}+\frac{\mathbf{w}_{2}}{\left\|\mathbf{w}_{2}\right\|}, \frac{b_{1}}{\left\|\mathbf{w}_{1}\right\|}+\frac{b_{2}}{\left\|\mathbf{w}_{2}\right\|}\right)
\end{aligned}
$$

We choose the angle bisector which has lower impurity. In our work we use gini index to measure impurity. Let $\tilde{\mathbf{w}}_{t}$ be a hyperplane which is used for dividing the set $S^{t}$ in two parts $S^{t_{l}}$ and $S^{t_{r}}$. Let $n_{+}^{t_{l}}$ and $n_{-}^{t_{l}}$ denote the number of patterns of the two classes in the set $S^{t_{l}}$ and $n_{+}^{t_{r}}$ and $n_{-}^{t_{r}}$ denote the number of patterns of the two classes in the set $S^{t_{r}}$. Then gini index of hyperplane $\tilde{\mathbf{w}}_{t}$ is given by,

$$
\operatorname{gini}\left(\tilde{\mathbf{w}}_{t}\right)=\frac{n^{t_{l}}}{n^{t}}\left[1-\left(\frac{n_{+}^{t_{l}}}{n^{t_{l}}}\right)^{2}-\left(\frac{n_{-}^{t_{l}}}{n^{t_{l}}}\right)^{2}\right]+\frac{n^{t_{r}}}{n^{t}}\left[1-\left(\frac{n_{+}^{t_{r}}}{n^{t_{r}}}\right)^{2}-\left(\frac{n_{-}^{t_{r}}}{n^{t_{r}}}\right)^{2}\right]
$$

where $n^{t}=n_{+}^{t}+n_{-}^{t}$ is the number of points in $S_{t}$. Also $n^{t_{l}}=n_{+}^{t_{l}}+n_{-}^{t_{l}}$ is the number of points falling in the set $S^{t_{l}}$ and $n^{t_{r}}=n_{+}^{t_{r}}+n_{-}^{t_{r}}$ is the number of points falling in the set $S^{t_{r}}$. We choose $\tilde{\mathbf{w}}_{3}$ or $\tilde{\mathbf{w}}_{4}$ to be the split rule for $S^{t}$ based on which of the two gives lesser value of gini index given by Eq. (8). Algorithm. 2 describes the complete decision tree learning algorithm.

\section{Experiments}

To test the effectiveness of Geometric DT we test its performance on several synthetic and real world data sets. We consider three synthetic problems 


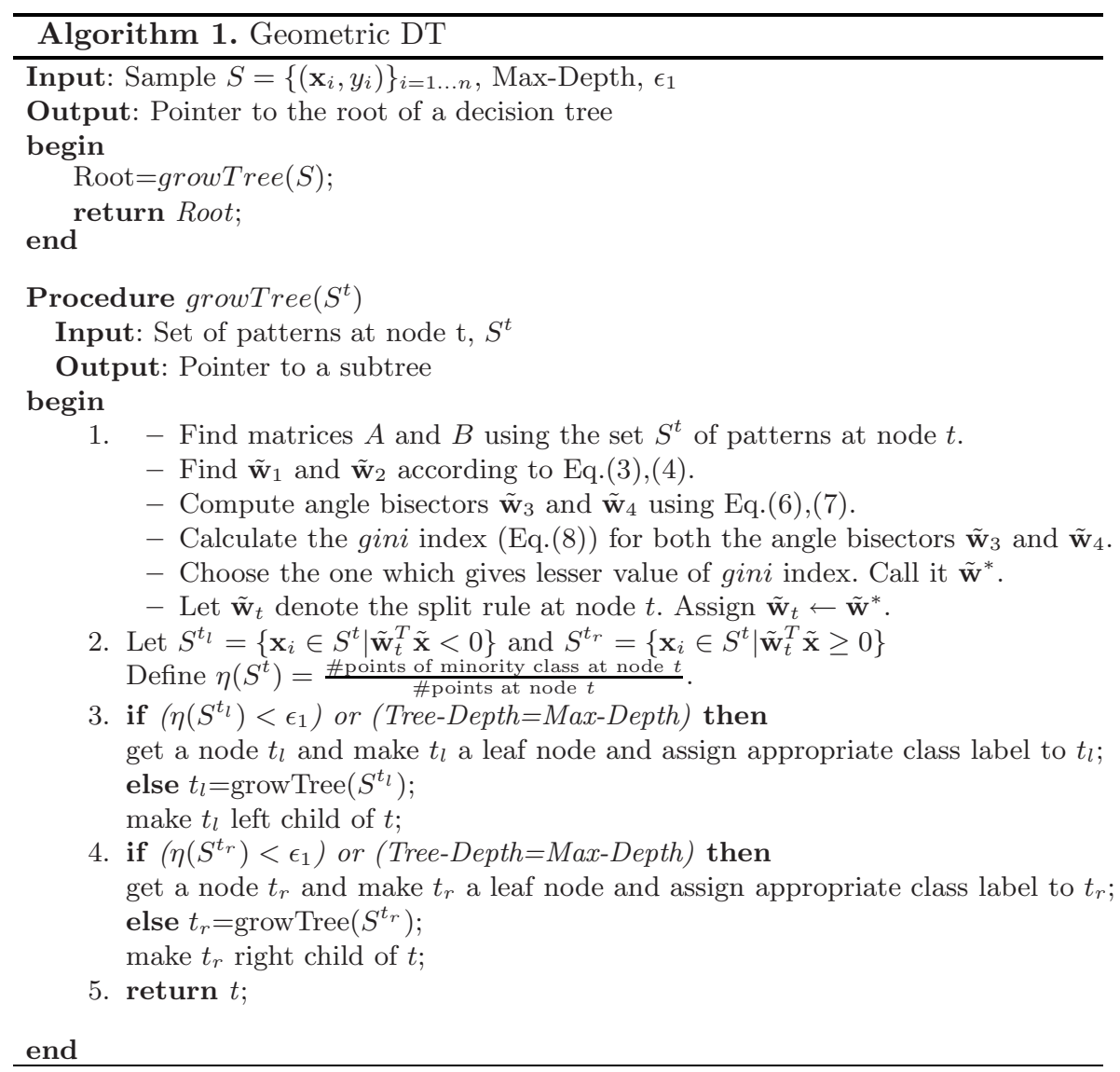

and two real world data sets from UCI ML repository [1. First two synthetic problems are rotated $2 \times 2$ and $4 \times 4$ checkerboard data sets in two dimensions. The third problem is in $\Re^{10}$. We sample points uniformly from $\left[\begin{array}{ll}-1 & 1\end{array}\right]^{10}$. Let $\tilde{\mathbf{w}}_{1}=[1,1,0,1,0,0,1,0,0,1,0]^{\prime} \tilde{\mathbf{w}}_{2}=[1,-1,0,0,1,0,0,1,0,0,0]^{\prime}$ and $\tilde{\mathbf{w}}_{3}=$ $[0,1,-1,0,-1,0,1,1,-1,1,0]^{\prime}$ be three hyperplane parameters in $\Re^{10}$. Now the points are labeled +1 or -1 based on the following rule

$$
y=\left\{\begin{array}{lll}
1, & \text { if } \quad\left(\tilde{\mathbf{w}}_{1}^{t} \tilde{\mathbf{x}} \geq 0 \& \tilde{\mathbf{w}}_{2}^{t} \tilde{\mathbf{x}} \geq 0 \& \tilde{\mathbf{w}}_{3}^{t} \tilde{\mathbf{x}} \geq 0\right) \| \\
& \left(\tilde{\mathbf{w}}_{1}^{t} \tilde{\mathbf{x}} \leq 0 \& \tilde{\mathbf{w}}_{2}^{t} \tilde{\mathbf{x}} \leq 0 \& \tilde{\mathbf{w}}_{3}^{t} \tilde{\mathbf{x}} \geq 0\right) \| \\
& \left(\tilde{\mathbf{w}}_{1}^{t} \tilde{\mathbf{x}} \leq 0 \& \tilde{\mathbf{w}}_{2}^{t} \tilde{\mathbf{x}} \geq 0 \& \tilde{\mathbf{w}}_{3}^{t} \tilde{\mathbf{x}} \leq 0\right) \| \\
& \left(\tilde{\mathbf{w}}_{1}^{t} \tilde{\mathbf{x}} \geq 0 \& \tilde{\mathbf{w}}_{2}^{t} \tilde{\mathbf{x}} \leq 0 \& \tilde{\mathbf{w}}_{3}^{t} \tilde{\mathbf{x}} \leq 0\right) \| \\
-1, \quad \text { else } &
\end{array}\right.
$$

For each synthetic problem 2000 points were sampled for training set and 1000 for testing set. To evaluate the performance of the algorithm on real dataset we use Breast Cancer Data set and Bupa Liver Disorder Data set [1]. Breast 
Cancer Data set is a 10 dimensional binary classification problem. It has total 683 instances, out of which we use 500 for training and 183 for testing. Bupa Liver Disorder Data set is a 6-dimensional binary classification problem. It has a total 345 instances, out of which we use 200 for training and 145 for testing. We compare performance of our approach with several algorithms, namely, CART-LC [2, OC1-AP, OC1(Oblique) 8], Support Vector Machine (SVM) 3] and GEPSVM 6]. For OC1 and CART-LC we use the downloadable [7] package. For SVM we use libsvm [4] code. Geometric DT is implemented in MATLAB. For GEPSVM also we wrote MATLAB code. We compare the performances on several measures like training time, training accuracy, test accuracy, number of leaves and depth of the tree. In the SVM method we used Gaussian kernel to capture non-linear classification boundaries. In case of GEPSVM also we used Gaussian kernel for all problems except the first problem where a pair of hyperplanes is good enough for classification. Best parameters for kernel were found by using 10-fold cross validation. The comparison results are shown in Table 1 (For OC1 and CART-LC which are randomized algorithms we show mean and standard deviation of results on 10 runs.) From Table. 1 we see that, over all the problems, Geometric DT performs better than all the other decision tree approaches in test accuracy, tree size and number of leaves. GEPSVM with linear kernel performs same as Geometric DT for the $2 \times 2$ checkerboard problem

Table 1. Comparison results on synthetic and real world data sets

\begin{tabular}{|c|c|c|c|c|c|c|c|}
\hline & & $\begin{array}{l}\text { Geometric } \\
\text { DT }\end{array}$ & OC1(AP) & $\begin{array}{l}\text { OC1 } \\
\text { (Oblique) }\end{array}$ & CART-LC & SVM & GEPSVM \\
\hline \multirow{5}{*}{\begin{tabular}{|l|}
$2 \times 2$ \\
Checker- \\
board
\end{tabular}} & $\operatorname{Tr} \operatorname{Time}(\mathrm{sec})$ & 0.064 & 0.031 & $3.112 \pm 0.83$ & $1.049 \pm 0.8$ & 0.132 & .004 \\
\hline & TrAcc & 99.70 & $99.34 \pm 0.3$ & $99.40 \pm 0.92$ & $98.57 \pm 1$ & 99.25 & 99.70 \\
\hline & TesAcc & 99.90 & $97.45 \pm 0.55$ & $98.52 \pm 1.17$ & $96.98 \pm 0.94$ & 99.5 & 99.90 \\
\hline & \# leaves & 4 & $39 \pm 5.83$ & $17.8 \pm 6.84$ & $22 \pm 8.1$ & - & - \\
\hline & Depth & 2 & $11.8 \pm 1.93$ & $8.4 \pm 3.24$ & $9.6 \pm 2.8$ & - & - \\
\hline \multirow{5}{*}{$\begin{array}{l}4 \times 4 \\
\text { Checker- } \\
\text { board }\end{array}$} & $\operatorname{TrTime}(\mathrm{sec})$ & 0.062 & 0.047 & $4.247 \pm 0.53$ & $2.029 \pm 0.98$ & 0.154 & 451.86 \\
\hline & TrAcc & 97.75 & $98.28 \pm 0.4$ & $\mathbf{9 8 . 7 8} \pm 0.76$ & $98.08 \pm 0.71$ & 98.10 & 90.70 \\
\hline & TesAcc & 98.50 & $92.48 \pm 0.86$ & $94.65 \pm 0.91$ & $92.53 \pm 0.9$ & 97.50 & 89.80 \\
\hline & \# leaves & 16 & $18.4 \pm 15.08$ & $82.7 \pm 21.96$ & $104.4 \pm 19.18$ & - & - \\
\hline & Depth & 4 & $16.3 \pm 2$ & $14.6 \pm 2.32$ & $15.4 \pm 2.46$ & - & - \\
\hline \multirow{5}{*}{$\begin{array}{l}10 \text { Dim- } \\
\text { ensional } \\
\text { Dataset }\end{array}$} & $\operatorname{TrTime}(\mathrm{sec})$ & 0.1192 & $0.249 \pm 0.02$ & $18.37 \pm 1.73$ & $31.54 \pm 9.67$ & 1.048 & 384.10 \\
\hline & TrAcc & 85.20 & $72.10 \pm 2.88$ & $74.76 \pm 3.64$ & $61.83 \pm 1.54$ & 98.65 & 92.20 \\
\hline & TesAcc & 84.60 & $60.40 \pm 1.86$ & $63.50 \pm 0.74$ & $61.29 \pm 1.46$ & 74.60 & 67.30 \\
\hline & \# leaves & 26 & $55.8 \pm 15.27$ & $31.7 \pm 12.81$ & $16.3 \pm 7.6$ & - & - \\
\hline & Depth & 7 & $11.3 \pm 1.57$ & $9.7 \pm 2.11$ & $7.9 \pm 1.79$ & - & - \\
\hline \multirow{5}{*}{$\begin{array}{l}\text { Breast } \\
\text { Cancer }\end{array}$} & $\operatorname{Tr} \operatorname{Time}(\mathrm{sec})$ & 0.0145 & $0.011 \pm 0.001$ & $1.41 \pm 0.42$ & $\mathbf{0 . 1 0} \pm 0.02$ & 0.061 & 9.63 \\
\hline & TrAcc & 96.4 & $97.30 \pm 1.03$ & $97.02 \pm 0.76$ & $95.14 \pm 1.65$ & 100 & 100 \\
\hline & TesAcc & 100 & $96.77 \pm 0.91$ & $97.27 \pm 1.15$ & $93.34 \pm 3.18$ & 77.05 & 77.05 \\
\hline & \# leaves & 3 & $7.9 \pm 4.68$ & $4.9 \pm 2.33$ & $\mathbf{2 . 8} \pm 1.32$ & - & - \\
\hline & Depth & 2 & $4 \pm 1.63$ & $2.9 \pm 1.29$ & $\mathbf{1 . 7} \pm 1.06$ & - & - \\
\hline \multirow{5}{*}{$\begin{array}{l}\text { Bupa } \\
\text { Liver } \\
\text { Disorder }\end{array}$} & $\operatorname{Tr} \operatorname{Time}(\mathrm{sec})$ & 0.0137 & 0.008 & $0.74 \pm 0.15$ & $0.06 \pm 0.01$ & 0.018 & 0.83 \\
\hline & TrAcc & 77 & $81.3 \pm 6.71$ & $84.2 \pm 5.15$ & $80.9 \pm 6.38$ & 100 & 79.00 \\
\hline & TesAcc & 72.41 & $59.3 \pm 2.28$ & $60.76 \pm 2.67$ & $59.66 \pm 2.33$ & 73.1 & 71.72 \\
\hline & \# leaves & 11 & $11.5 \pm 6.45$ & $12.9 \pm 5.51$ & $12.5 \pm 7.09$ & - & - \\
\hline & Depth & 7 & $7 \pm 3.13$ & $7.6 \pm 3.17$ & $6.7 \pm 3.13$ & - & - \\
\hline
\end{tabular}


because for this problem the two approaches work in a similar way. But when there are more than two hyperplanes required, GEPSVM with Gaussian kernel performs worse than our decision tree approach. For example, for $4 \times 4$ checkerboard example, GEPSVM can achieve only about $89.8 \%$ test accuracy while our decision tree gives about $98.5 \%$ test accuracy. Moreover, with Gaussian kernel, GEPSVM solves the generalized eigenvalue problem of the size of number of points, whereas our decision tree solves the generalized eigenvalue problem of the dimension of the data (which is the case with GEPSVM only when it uses linear kernel). This gives us an extra advantage in computational cost over GEPSVM.

In 10-dimensional problem, both in test accuracy and training time, Geometric DT performs better than all the other approaches. This shows that our algorithm scales with data dimension. For the real world problems also Geometric DT performs well. It gives highest test accuracy with Breast Cancer Data set. For Liver Disorder Data set also it performs better than all other decision tree approaches and the test accuracy of SVM is marginally better.

In Figure. 1 we show the effectiveness of our algorithm in terms of capturing the geometric structure of the classification problem. We show the first few hyperplanes generated by our approach and OC1(oblique) for $2 \times 2$ and $4 \times 4$ checkerboard data. We see that our approach learns the correct geometric structure of the classification boundary, whereas the OC1(oblique), which uses the gini index as impurity measure, does not capture that.

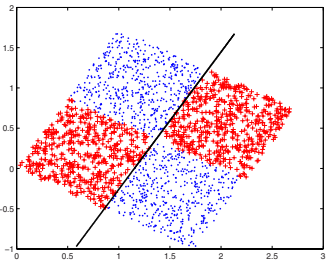

(a)

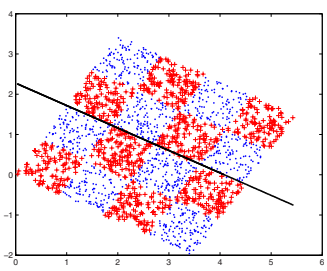

(d)

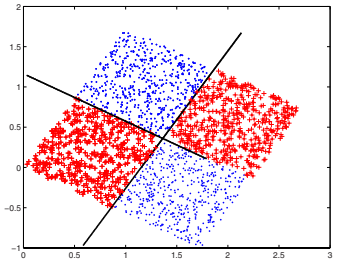

(b)

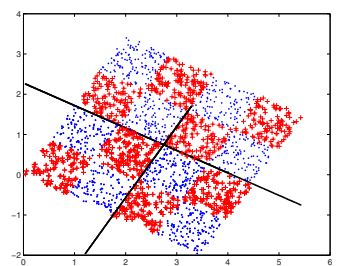

(e)

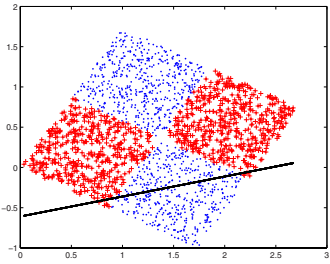

(c)

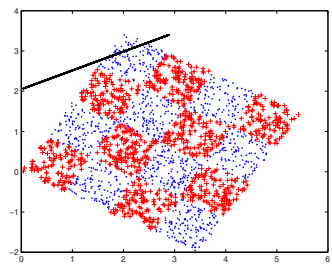

(f)

Fig. 1. Comparisons of Geometric DT with OC1(Oblique) on rotated $2 \times 2$ and $4 \times 4$ checkerboard data: (a)Hyperplane at root node learnt using Geometric DT on rotated $2 \times 2$ checkerboard data; (b) Hyperplane at left child of root node learnt using Geometric DT on rotated $2 \times 2$ checkerboard data; (c)Hyperplane at root node learnt using OC1(Oblique) DT on rotated $2 \times 2$ checkerboard data; (d), (e) and (f) show the hyperplanes for $4 \times 4$ checkerboard data in a similar way 


\section{Conclusions}

In this paper we presented a new algorithm for learning oblique decision trees. The novelty is in learning hyperplane that captures the geometric structure of the data using the GEPSVM idea. Through empirical studies we showed that the method performs better than the other approaches and also captures the geometric structure well. Though we consider only the 2-class problems here, the approach can easily be generalized to multiclass problems by learning a split rule, at each node, to separate the majority class from the rest. We will be explaining this in our future work.

\section{References}

1. Asuncion, A., Newman, D.J.: UCI machine learning repository (2007)

2. Breiman, L., Friedman, J.H., Olshen, R.A., Stone, C.J.: Classification and Regression Trees. Wadsworth and Brooks (1984)

3. Burges, C.J.C.: A tutorial on support vector machines for pattern recognition. In: Knowledge Discovery and Data Mining, vol. 2, pp. 121-167 (1998)

4. Chang, C.-C., Lin, C.-J.: LIBSVM: a library for support vector machines (2001), http://www.csie.ntu.edu.tw/ cjlin/libsvm

5. Fung, G., Mangasarian, O.L.: Proximal support vector machine classifiers. In: Knowledge Discovery and Data Mining (KDD), pp. 77-86 (2001)

6. Mangasarian, O.L., Wild, E.W.: Multisurface proximal support vector machine classification via generalized eigenvalues. IEEE Transaction on Pattern Analysis and Machine Intelligence 28(1), 69-74 (2006)

7. Murthy, S.K., Kasif, S., Salzberg, S.: The OC1 decision tree software system (1993), http://www.cs.jhu.edu/ salzberg/announce-oc1.html

8. Murthy, S.K., Kasif, S., Salzberg, S.: A system for induction of oblique decision trees. Journal of Artificial Intelligence Research 2, 1-32 (1994) 